\title{
Necrotizing pneumonia caused by Streptococcus pneumoniae serotype 3 despite PCV13
}

\author{
Gülsüm Alkan, M.D. ${ }^{a}$, Melike Emiroğlu, Assist. Prof. M.D. ${ }^{a}$, Hatice T. Dă̆ı, Assoc. Prof. M.D. ${ }^{b}$, \\ Venhar Gürbüz, Biologistc and Mehmet Ceyhan, Prof. M.D. ${ }^{c}$
}

\begin{abstract}
Streptococcus pneumoniae is the most common cause of complicated pneumonia. Pneumococcal necrotizing pneumonia (PNP) is a rare and serotype related complication. Serotypes $1,3,14,15,19 \mathrm{~A}$ and 33 were the most reported serotypes in children with PNP before immunization. Despite widespread vaccination, S. pneumoniae is still cause of invasive diseases. We reported a child, fully immunized with 13-valent conjugated pneumococcal vaccine (PCV13) who was diagnosed PNP due to serotype 3 . Breakthrough invasive infection caused by $S$. pneumoniae must be considered in mind despite fully vaccination.

Key words: Streptococcus pneumoniae, necrotizing pneumonia, immunization, child.
\end{abstract}

http: / / dx.doi.org/10.5546/ aap.2019.eng.e155

To cite: Alkan G, Emiroğlu M, Dağ HT, Gürbüz V, Ceyhan M . Necrotizing pneumonia caused by Streptococcus pneumoniae serotype 3 despite PCV13. Arch Argent Pediatr 2019;117(2):e155-e157.

\section{INTRODUCTION}

S. pneumoniae is the most common cause of invasive diseases, such as pneumonia, meningitis, bacteremia and sepsis in children. To date 91 different capsular types have been identified on basis of capsular polysaccharide. Different serotypes exhibit differences in clinical expression of the disease. About 13 serotypes are responsible for $>75 \%$ of invasive pneumococcal diseases (IPD) in children. ${ }^{1}$ Conjugated pneumococcal

a Selcuk University Faculty of Medicine, Department of Pediatric Infectious Diseases, Konya, Turkey.

b. Selcuk University Faculty of Medicine, Department of Medical Microbiology, Konya, Turkey.

c. Hacettepe University Pediatric Infectious Diseases Department, Ankara, Turkey.

E-mail address:

Gülsüm Alkan, M.D.: galkan-85@hotmail.com

Funding: None.

Conflict of interest: None.

Received: 5-5-2018

Accepted: 10-25-2018 vaccine 7 (PCV7) which include $4,6 \mathrm{~B}, 9 \mathrm{~V}, 14,18 \mathrm{C}$, 19F, 23F serotypes, was introduced to Turkish national immunization program in 2009 and then was replaced by PCV13 which includes 1, 3, 5, 6A, 7F and 19A serotypes. ${ }^{2}$ In a multicenter study from Turkey, the potential serotype coverages of PCV-7, PCV-10 and, PCV-13 were $16.3 \%$, $45.4 \%$, and $60 \%$, respectively, for pediatric parapneumonic empyema. ${ }^{3}$ By the end of 2015, the vaccination rate for PCV13 was reported as $97 \%$ in Turkey. Despite high vaccination rates and strong immunoreaction to conjugate vaccines, life-threatening complications such as pneumococcal necrotizing pneumonia (PNP) or empyema still occur.

\section{CASE REPORT}

A previously healthy 20-month-old boy was admitted to hospital with a 3 days history of cough and fever. His medical and family history was unremarkable. He had been vaccinated with PCV13 at 2, 4, 6 and 12 months of age. On physical examination his body temperature was $39^{\circ} \mathrm{C}$, pulse rate was 160 beats / min and respiratory rate was 56 breaths/min. His weight and height were $13 \mathrm{~kg}$ (percentile $75 \%$ ) and $90 \mathrm{~cm}$ (percentile $90 \%$ ), respectively. The physical examination does not show suggestive findings of a determined syndromic picture. Decreased breath sounds were noted over the left lung field. The result of other system examinations was normal.

Laboratory data: white blood cells count $14,6 \times 10^{3} / \mathrm{mm}^{3}$, neutrophils $65 \%$, lymphocytes $10 \%$, hemoglobin $10 \mathrm{~g} / \mathrm{dl}$, platelets $179000 / \mathrm{mm}^{3}$, erythrocyte sedimentation rate $31 \mathrm{~mm} / \mathrm{hr}, \mathrm{C}$-reactive protein $42 \mathrm{mg} / \mathrm{dl}$, biochemical parameters were normal. Chest X-ray showed total opacity of the left lung. Thorax tomography (CT) showed extensive consolidation with necrotizing pneumonitis in the left lower lobe and moderate left pleural effusion (Figure 1). Thoracic drainage tube was inserted and, about $250 \mathrm{~mL}$ purulent fluid drained. Gram positive diplococci were seen on gram stain. Intravenous ampicillin-sulbactam (200 mg/ kg/day) and clindamycin (40 mg/ 
$\mathrm{kg}$ /day) were started. Penicillin-susceptible $S$. pneumoniae grew in empyema culture. Isolate was identified as serotype 3 by the Quellung reaction using serotype-specific antisera (Staten's Serum Institute, Copenhagen, Denmark). Fever was resolved within 2 days, but despite the presence of a drainage tube, respiratory distress and pleural effusion were not resolved and, left lung did not expand on chest $x$-ray. On the 30 th day of treatment, pleural thickening as $4 \mathrm{~mm}$ with fully atelectatic left lung and thick pleural effusion were detected by thorax tomography (CT). After left pleural decortication was performed, left lung expanded rapidly (Figure 2). Immunologic screening tests (blood cell counts, peripheral blood lymphocyte subsets, serum total immunoglobulin levels, dihydrorhodamine test, complement system) were all in normal limits, and ELISA for human immunodeficiency virus was negative. Spleen was seen normal by ultrasound, Howell-Jolly bodies was not detected on peripheral smear. The total pneumococcal IgG titer was $2.2 \mathrm{microgr} / \mathrm{ml}$. Antibiotic treatment was completed for 6 week and the patient was discharged. The patient has been followed up for 1 year without sequelae.

\section{DISCUSSION}

Necrotizing pneumonia is characterized by destruction of the lung parenchyma resulting in multiple cavities and is often accompanied with empyema. Generally, PNP is diagnosed by serial radiography and thoracic CT. Staphylococcus aureus, Streptococcus pneumoniae, and Klebsiella pneumoniae are the most common cause of PNP.
Surgical resection is indicated in cases of sepsis, persistent fever, empyema or refractory to antibiotic treatment. ${ }^{1,-5}$

S. pneumoniae serotypes such as $1,3,14,15$, $19 \mathrm{~A}$ and 33 were the most reported serotypes in children with PNP before immunization, especially serotype 3 was more likely to cause it. ${ }^{6}$ Although the mechanism of necrosis for serotype 3 is not clear, it has been hypothesized to be related with rapid accumulation of capsular polysaccharides leading to a large antigenic load and possible reduced humoral immune responses. ${ }^{7}$

Despite widespread vaccination, reports of vaccine failure from PCV13 (included serotype 3) have been documented for parapneumonic effusion. Antachopoulos et al. reported the failure of PCV13 serotype 3 vaccine with complicated pneumonia from Greek. They reported 5 children with empyema due to serotype 3 , although they were fully immunized with PCV13. Patients were treated with antibiotic, chest tube and fibrinolysis. None of them required surgery. Multivariate vaccines cannot protective at the same level in every serotype. In some studies, pneumococcal polysaccharide IgG responses for serotype 3 before and after toddler dose were lower than other serotypes. ${ }^{8,9}$

The greatest spread of pneumococci to the lungs comes from the oropharynx. Bacteremic pneumonia accounts for about $12-16 \%$ of invasive pneumococcal disease among children 2 years old or younger. ${ }^{10} \mathrm{PCV}$ is particularly effective in infections with bacteremia. Low protection of vaccine in complicated non-bacteremic
Figure 1. Thorax CT: Extensive consolidation with necrotizing pneumonitis and moderate left pleural effusion

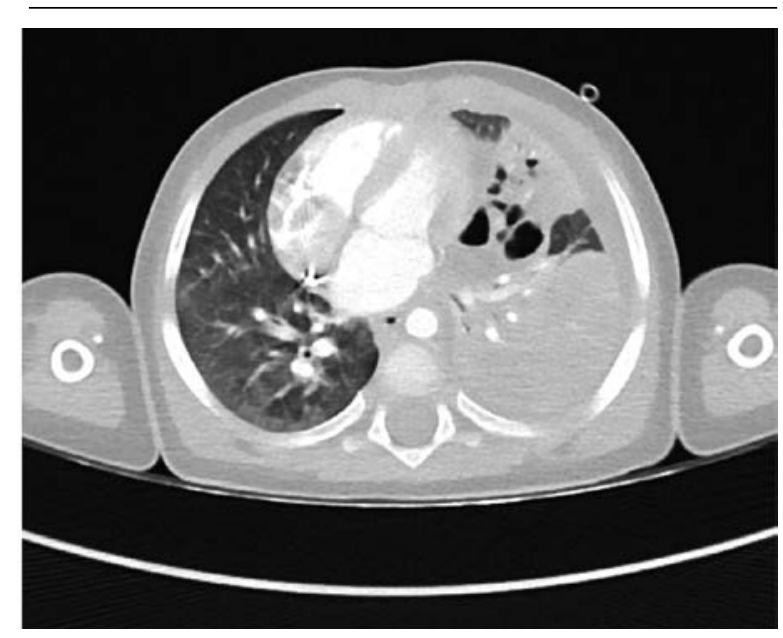

FIGURE 2. Chest X-ray: Left lung expanded after decortication

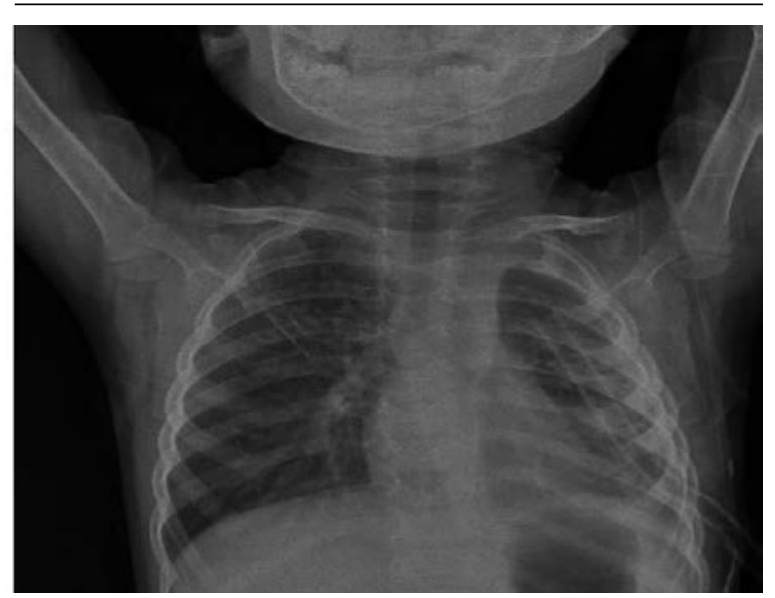


pneumonia can be related with this condition. The patient blood culture was sterile, despite no antibiotic use. On the other hand, pneumococcal antibiotic resistance is a worldwide challenge of disease. Antimicrobial resistance causes different clinical presentation of disease, making it more difficult to diagnose and to treat. ${ }^{11}$ However, our patient's culture susceptible to penicillin, the clinical response was inadequate to therapy. Antibiotic resistance, and immunodeficiency should be kept in mind on complicated cases. Primary immunodeficiency known to underlie clinical disease caused by S. pneumoniae, include congenital asplenia, complement deficiency, antibody deficiency, mutations of NEMO, IRAK4 or MYD88 genes. The patient immunologic screening tests was normal. Clinical infectious phenotype of IRAK-4 and MyD88 deficiencies are extremely severe. Laboratory (CRP concentration, total leukocyte counts, and neutrophil numbers) and clinical (temperature) signs of inflammation are typically low even in such infections..$^{12}$ The patient's clinic and laboratory features were not compatible with IRAK-4 or MyD88 deficiencies.

According to our knowledge, a case of PNP in a child fully immunized has not been reported in literature. The patient total pneumococcal Ig $\mathrm{G}$ was positive but, unfortunately, we could not measure the serotype-specific antibody levels. We cannot define this case a serotype specific vaccine failure.

PNP has required longer duration of treatment, pleural drainage and surgery. Irrespective of high vaccination rate, pediatricians should be alert to possible complications of IPD. Early definition of Streptococcus pneumoniae serotypes can be useful for treatment approach. Continuing active surveillance is important for determination of vaccine failure and design of new vaccines.

\section{REFERENCES}

1. Lynch JP 3rd, Zhanel G.G. Streptococcus pneumoniae: epidemiology, risk factors, and strategies for prevention. Semin Respir Crit Care Med. 2009; 30(2):189-209.

2. Ceyhan M, Ozsurekci Y, Gürler N, Öksüz L, et al. Serotype distribution of Streptococcus pneumoniae in children with invasive diseases in Turkey: 2008-2014. Hum Vaccin Immunother. 2016; 12(2):308-13.

3. Ceyhan M, Ozsurekci Y, Gürler N, Özkan S, et al. Distribution of Streptococcus pneumoniae serotypes that cause parapneumonic empyema in Turkey. Clin Vaccine Immunol. 2013; 20(7):972-6.

4. Masters IB, Isles AF, Grimwood K. Necrotizing pneumonia: an emerging problem in children? Pneumonia (Nathan). 2017; 9:11.

5. LuS, TsaiJD, Tsao TF, Liao PF, et al. Necrotizing pneumonia and acute purulent pericarditis caused by Streptococcus pneumoniae serotype 19A in a healthy 4-year old girl after one catch-up dose of 13-valent pneumococcal conjugate vaccine. Paediatr Int Child Health. 2016; 36(3):235-9.

6. Tsai YF, Ku YH. Necrotizing pneumonia: a rarecomplication of pneumonia requiring special consideration. Curr Opin Pulm Med. 2012; 18(3):246-52.

7. Bender JM, Ampofo K, Korgenski K, Daly J, et al. Pneumococcal necrotizing pneumonia in Utah: does serotype matter? Clin Infect Dis. 2008; 46(9):1346-52.

8. Diawara I, Zerouali K, Elmdaghri N, Abid A. A case report of parapneumonic pleural effusion caused by Streptococcus pneumoniae serotype 19A in a child immunized with 13-valent conjugate pneumococcal vaccine. BMC Pediatr. 2017; 17 (1):114.

9. Antachopoulos C, Tsolia MN, Tzanakaki G, Xirogianni $A$, et al. Parapneumonic pleural effusions caused by Streptococcus pneumoniae serotype 3 in children immunized with 13-valent conjugated pneumococcal vaccine. Pediatr Infect Dis J. 2014; 33(1):81-3.

10. Centers of Disease Control and Prevention. Pneumococcal Diseases. Clinical Features. Atlanta, 2017. [Accessed on: Nov 6, 2018]. Available at: https://www.cdc.gov/ pneumococcal/clinicians / clinical-features.html

11. Tanır Basranoglu S, Karadag Oncel E, Aykaç K, Ozsurekci $\mathrm{Y}$, et al. Invasive pneumococcal disease: from a tertiary care hospital in the post-vaccine era. Hum Vaccin Immunother. 2017; 13(4):962-4.

12. Picard C, von Bernuth H, Ghandil P, Chrabieh M, et al. Clinical features and outcome of patients with IRAK-4 and MyD88 deficiency. Medicine (Baltimore). 2010; 89(6):403-25. 